\title{
DESIGN FOR MONITORING OF A RESEARCH VEHICLE
}

\author{
Kukurowski, Nobert (1); Stetter, Ralf (2); Witczak, Marcin (1) \\ 1: Institute of Control and Computation Engineering, University of Zielona Góra; 2: University of \\ Applied Sciences Ravensburg-Weingarten
}

\begin{abstract}
In recent years, more and more technical systems dispose of some form of intelligence which allows to control and diagnose the processes and states in such systems. One important prerequisite for both control and diagnosis is monitoring, i.e. a systematic observation, surveillance or recording of entities of a technical product or its surroundings by any technical means. Current investigations were able to clarify that the design of a technical system can ease the control and diagnosis of this system. The same is true for monitoring; this fact will be demonstrated in this paper on the example of a research vehicle which is intended to foster the development of mapping systems and algorithms. In a sense, the main objective of this vehicle is also a kind of monitoring. Due to its unique design, this vehicle is able to navigate on all kind of terrains. It is equipped with several forms of sensors, which are consciously mounted at certain positions on the vehicle in order to allow a detailed detection of the surroundings. The investigation, how design can ease monitoring, was supported by a well-known model of product concretization and concrete insights could be found on all levels.
\end{abstract}

Keywords: Design for X (DfX), Systems Engineering (SE), Mechatronics

\section{Contact:}

Stetter, Ralf

University of Applied Sciences Ravensburg-Weingarten

Mechanical Engineering

Germany

ralf.stetter@hs-weingarten.de 


\section{INTRODUCTION}

In recent years, more and more technical systems dispose of some form of intelligence which allows to control and diagnose the processes and states in such systems. One important prerequisite for both control and diagnosis is monitoring, i. e. a systematic observation, surveillance or recording of entities of a technical product or its surroundings by any technical means. Current investigations were able to clarify that the design of a technical system can ease the control and diagnosis of this system. The same is true for monitoring; this fact will be demonstrated in this paper on the example of a research vehicle which is intended to foster the development of mapping systems and algorithms. In a sense, the main objective of this vehicle is also a kind of monitoring. Due to its unique design, this vehicle is able to navigate on all kind of terrains. It is equipped with several forms of sensors, which are consciously mounted at certain positions on the vehicle in order to allow a detailed detection of the surroundings. The central research objective was the development of concrete design guidelines. The investigation, how design can ease monitoring, was supported by a well-known model of product concretization. On all levels of this model, concrete insights for the Design for Monitoring could be formulated. The paper is concluded with a monitoring application example - the mapping of a room with the research vehicle. In the following section, a detailed background is given and a model of product concretization is presented which will serve as a structure for the subsequent investigations. The objectives and design of the research vehicle are explained in Section 3. In Section 4 the possibilities for Design for Monitoring are investigated on the different levels of product concretization. Section 5 presents an application example the mapping of a room. Section 6 summarizes the results and proposes directions for further research.

\section{BACKGROUND}

For describing the functionality of intelligent entities in technical systems, the notions control, monitoring and diagnosis can be distinguished on an abstract level. The notion "control" stands for activities with the objective to manage, command, direct or regulate the behaviour of devices or systems; control systems are present in technical systems since many decades and were the object of numerous scientific investigations and developments. One notable extension is adaptive control, which is based on an aggregation of conventional control with some form of recursive system identification (Astrom and Wittenmark (2009)). The core meaning of the notion is the process of estimating the condition of certain entities. To be more precise, in technical applications the term diagnosis describes activities which aim at detecting and identifying faults. Diagnosis is a crucial building block for safer and more reliable technical systems which are also easier to maintain. Numerous scientific investigations have covered this field in the last three decades and have delivered striking research results (Blanke et al. (2016); Isermann (2006); Korbicz et al. (2012)). Concerning the design of technical systems the main function of diagnosis can be described as "detecting and identifying product or process abnormalities". In the last decades the functionality of diagnosis was combined with control functionalities leading to fault-tolerant control (Witczak (2014)). In the scope of product design, the notion "monitoring" summarizes all kinds of systematic observation, surveillance or recording of entities of a technical system or its surroundings by any technical means (compare Stetter (2017)). The entities of a technical system can be product characteristics (e. g. drive motor temperature), product flows (e. g. electrical flow), product position and orientation, product performance (e. g. speed) and product mission fulfilment (e. g. mapped surface area - compare Figure 1). The purposes of monitoring are manifold and can include control tasks (e. g. speed control) or diagnosis tasks (e. g. fault accommodation), but also high level control and diagnosis in superordinate systems such as the central mission control system of the research vehicle. Furthermore, the purpose of monitoring can be connected with the desire to provide. On the other hand the purpose of product monitoring may be derived (amongst others) from a need or a desire for high level control and diagnosis (e. g. manufacturing execution systems) or for top level control and planning (e. g. enterprise resource planning) or human machine interaction (e. g. instrumentation panels). A combination of a system for control and monitoring is frequently referred to as "Supervisory Control and Data Acquisition" (SCADA) system (compare Cherdantseva et al. (2016)). SCADA systems are used for controlling and monitoring complex infrastructures such as electric power grids, transportation systems, communication networks, gas pipelines and water distribution networks (Van Long et al. (2015)). Frequently SCADA systems also dispose of diagnosis functionalities. 


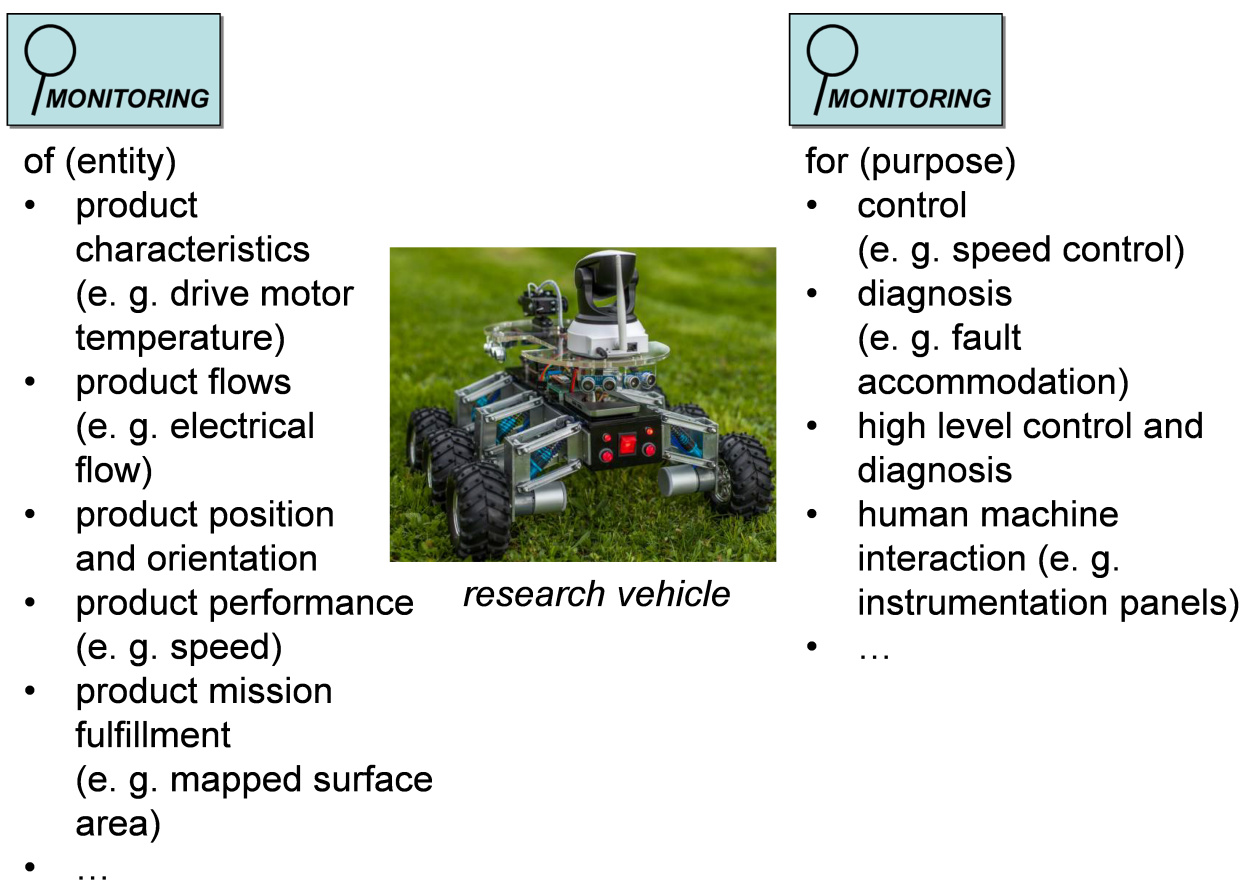

Figure 1. Forms and purposes of monitoring (in the scope of product design)

The next generation of technical systems with intelligence will require an effective and efficient design, development and management of the monitoring functionalities. Algorithms, strategies, methods and tools which address the issues of monitoring and address the integration in technical systems are consequently very important for design; their development can be systematized and fostered by means of a product concretization model. In recent years, several models of product concretization were proposed (Ponn and Lindemann (2011); Pfenning et al. (2017); Eigner et al. (2018)). Their essence can be reflected in a rather mundane model of product concretization which is shown in Figure 2.

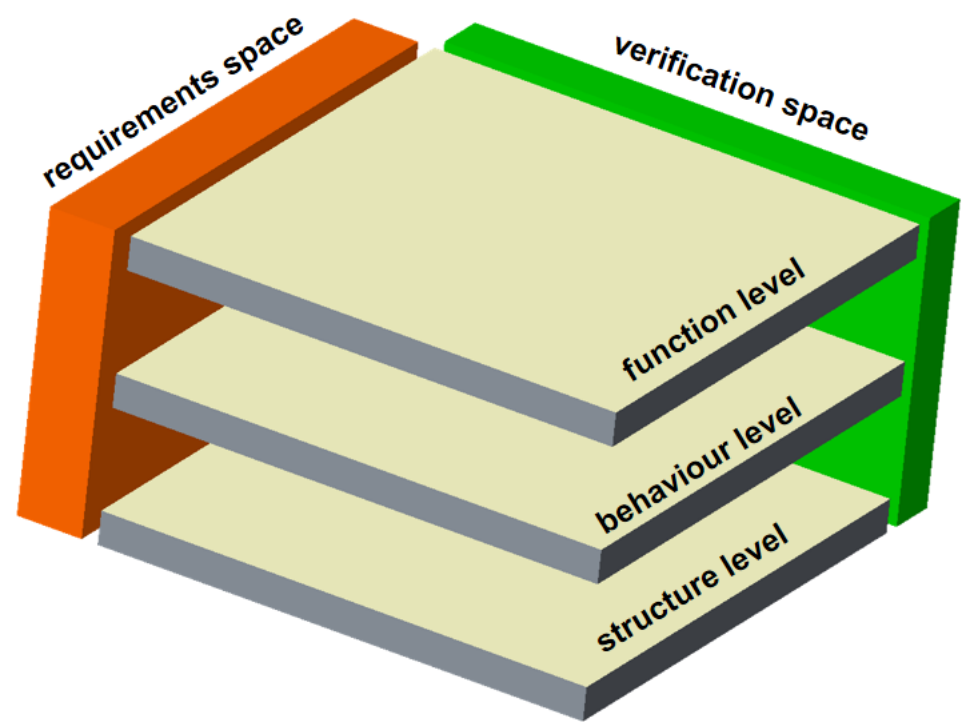

Figure 2. Model of product concretization

Three levels form the core of this model. The function level serves to generate a solution-neutral description of the system functionality in the form of a functional system architecture. The choice of the concept for a physical realization is performed on the behaviour level. While the essential principles are determined on this level, the concrete geometry and material are developed on the structure level, which is the most concrete level. Visible is also an orthogonal positioning of the requirements space and the verification space, indicating that those aspects are present on all levels and in all phases of the design process. This model is used to structure the investigation in Section 4. 


\section{DESIGN OF A RESEARCH VEHICLE}

The investigation in Section 4 is based on an exemplary technical system - research vehicle which is intended to foster the development of mapping systems and algorithms. The central target of this vehicle is also a kind of monitoring. The vehicle disposes six independent wheels, each of which is driven by its own electrical motor. The whole suspension is movable. Two shock absorbers are mounted on each wheel, which can be adjusted. Thanks to this suspension, AGV is adapted to work in difficult terrain. The research vehicle is shown in different drive scenarios in Figure 3.
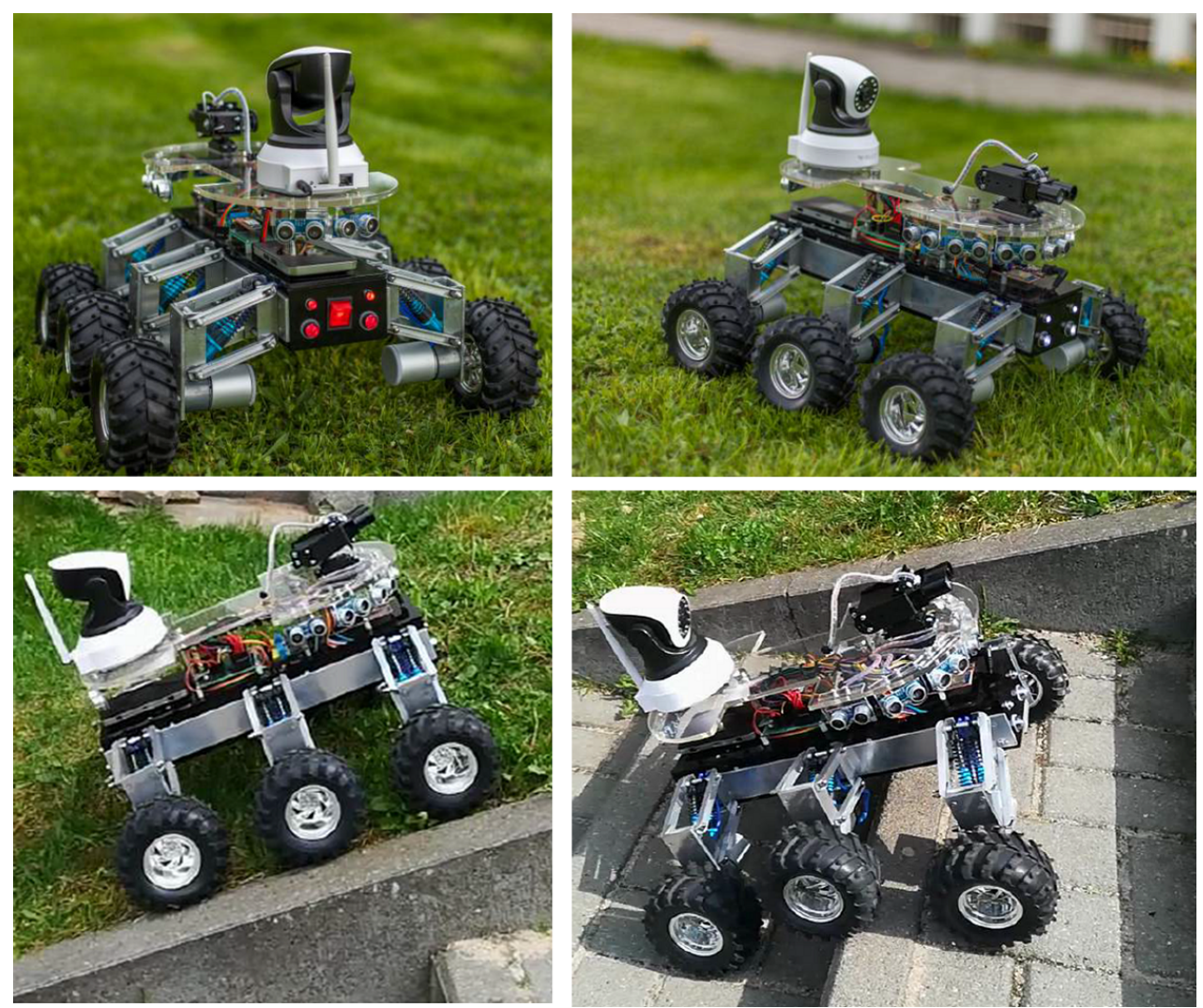

Figure 3. Research vehicle in different drive scenarios

Due to its unique design, this vehicle is able to navigate on all kind of terrains. This quality is fostered by a double wishbone design of the chassis (compare Figure 4). The complete chassis is shown in a situation with no load on the wheels on the left side of Figure 4. The sprung situation of one side of the chassis is shown on the right side of Figure 4.

The vehicle is equipped with several forms of sensors, which are consciously mounted at certain positions on the vehicle in order to allow a detailed detection of the surroundings. The research vehicle
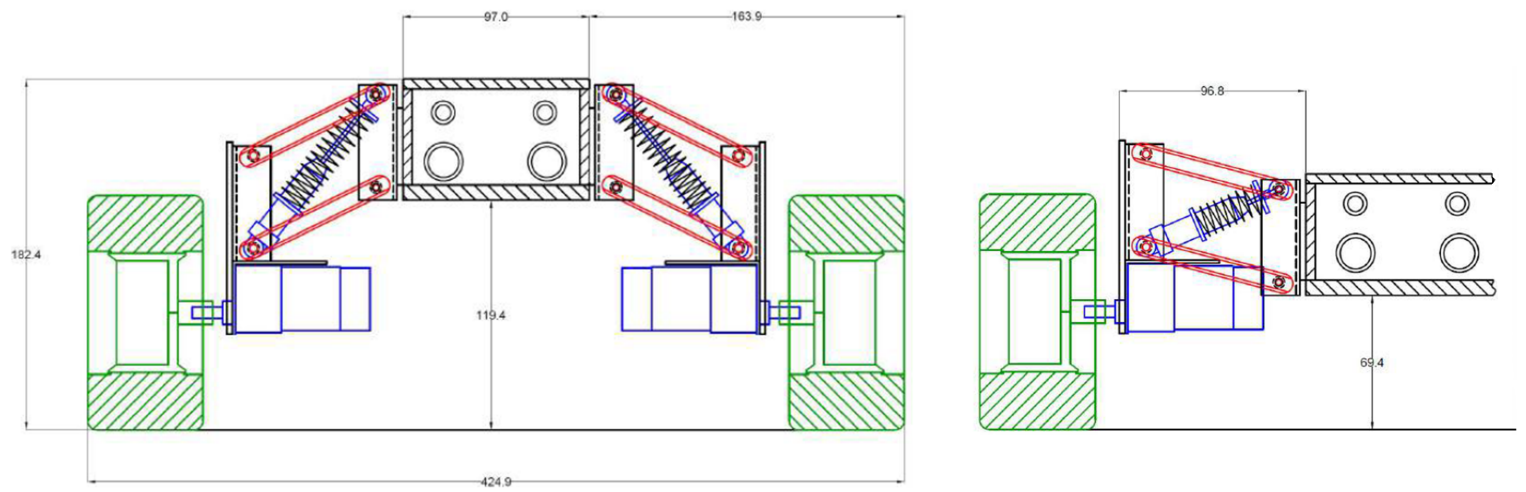

Figure 4. Chassis of the research vehicle 
disposes of ultrasonic sensors to avoid obstacles, a LIDAR sensor (Light Detection And Ranging - a radar-related method for optical distance measurement) to scan the room, and a optical Wi-Fi camera installed to view the image from the AGV in real time, this camera can be rotated ( 355 degrees) and swiveled. Obviously, some form of redundancy and sensor overlap (compare Stetter (2019)) is present in this design. A special focus will be on these sensors, but also on the other sensors on the vehicle (sensors in the wheels for odometry), for generating insights concerning Design for Monitoring.

\section{POSSIBILITIES FOR DESIGN FOR MONITORING}

This section describes insights concerning the Design for Monitoring; the structure of the sub-section corresponds to the model of product concretization shown in Figure 2.

\subsection{Requirement space}

The requirements space is crucial, because requirements are a decisive factor in industrial product and mechatronic design (compare Carryer et al. (2010), Holder et al. (2017)). Four of ten top risks in projects are linked to requirements (Hruschka (2019)). Sometimes monitoring activities are not considered as essential for the main function of a product. However, in the application example the main function is a kind of monitoring. Similar to many other technical systems the vehicle also requires monitoring for an economic and ecological performance. Thus, it can be assumed that monitoring functionalities should also be treated as immediate requirements and not only as means which help to fulfil other requirements. Monitoring functions are frequently linked to the direct functionality of the product. For an example, a monitoring function "detect low battery status" of the battery of the research vehicle is connected to the direct function "supply energy". Consequently, for transparent design processes it is advantageous if requirements concerning monitoring functions can be associated to requirements describing the (direct) functionality which should be monitored. A central method concerning the requirements space can be the "Failure Mode and Effects Analysis" (FMEA). It allows identifying necessities for diagnosis (compare Stetter and Phleps (2011)). Frequently, the required diagnosis function relies on sensor information and consequently leads to requirements concerning functions. To conclude, the following insights appear concerning Design for Monitoring in the requirement space:

- $\quad$ it is sensible to formulate necessary monitoring functionalities as immediate requirements,

- it is sensible to associate requirements concerning monitoring with requirements concerning direct functions,

- $\quad$ FMEA can be applied for identifying necessary diagnostic functions which will lead to monitoring requirements.

\subsection{Function level}

Frequently, necessary functions of future technical systems are described in requirements. Function models allow to describe (on an abstract level) how these functions can be realized; several possibilities are described in literature, a good overview is given by Eisenbart et al. (2017). The function model, proposed by Ehrlenspiel and Meerkamm (2013), offers the advantageous possibility to include states and to describe the links between secondary flows and primary flows (types: condition state, process state, additional state). Figure 5 contains the notation (left side) and an example of the flow-orientated function model. In the example of the research vehicle (right side of Figure 5) the quality that the vehicle is moving is linked in form of a "process state (P)" to the main flow - denoting an outcome ("product") of the operation in the main flow which can be important for monitoring purposes. This type of function model can illustrate which entities are being monitored, may clarify the accessibility of the main flows and may localize the monitoring function on a functional level. Possible monitoring operations could test, if the input states of a function or condition states are existing.

In contrast to flow-oriented function models, other function models concentrate on relations such as the one proposed in connection with the tools of the "Theory of Inventive Problem Solving" (TIPS compare e. g. Livotov and Petrov (2011)). A notation overview and an example describing the research vehicle is shown in Figure 6.

Similar to flow-oriented function models, the relation-oriented function models may foster a deeper understanding of the monitoring functions and the associations between them. The analysis of these associations can lead to an understanding of the underlying causes. Both types of function models 

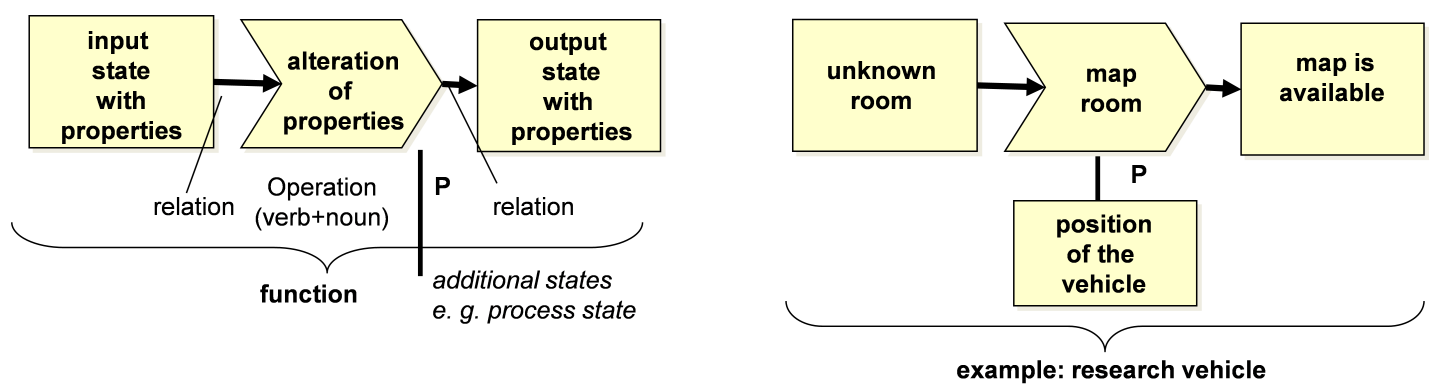

Figure 5. Flow-oriented function model

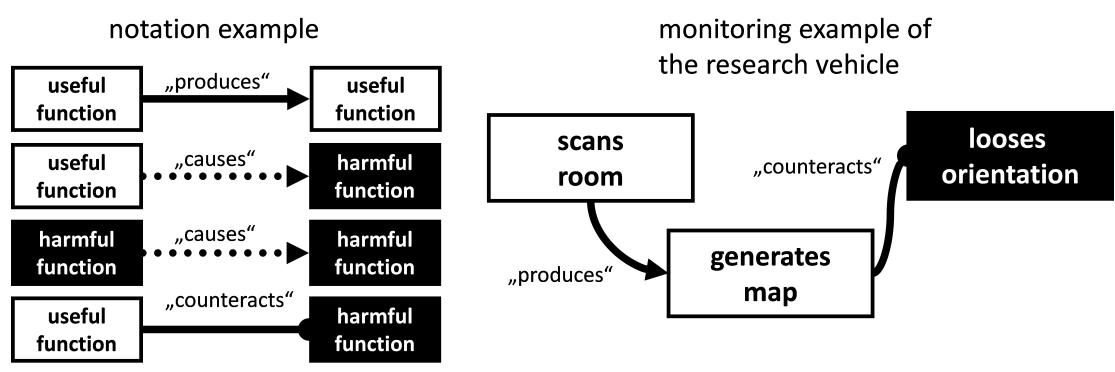

Figure 6. Relation-oriented function model

support the understanding and analysis of sensor functionality. A promising approach for an abstract analysis was also developed in the field of control engineering. The so-called "structural model" is also an abstraction of the behavioural model of a dynamic system Blanke et al. (2016). The focus of the structural model is on the structure of constraints which describe the dynamical behaviour of the system. The main goal of the application of this structural model is the identification of fault diagnosis possibilities by means of analytic redundancy relations, i. e. to which extend can faults be detected, if an elaborate control system disposes of a mathematical model and compares the results of this model with current measurements. The results of this comparison are called residuals and are a core element in Fault Diagnosis and Identification (FDI). Main outcomes of the analysis of the structural model can be Blanke et al. (2016):

- a system is structurally diagnosable or monitorable, if it can be tested whether the system constraints are satisfied,

- certain characteristics of the signature matrix allow the determine whether a possible fault is detectable (the presence of the fault can be detected) or even isolable (it can be determined that exactly this fault is present),

- a system is structurally observable, if all unknown variables can be determined from the known variables.

This outcomes can be used in an early and abstract phase of design, which degree of fault diagnosis the present monitoring functions will allow; the benefits could be shown by Frisk and Krysander (2008).

To conclude, the following insights appear concerning Design for Monitoring in the function level:

- flow-oriented function models with explicit links between main flows and auxiliary flows and relation-oriented function models may enhance the functional transparency and foster the understanding of the deeper relationships between direct and monitoring functions,

- function models can clarify the main flows which can be essential for monitoring functionality,

- structural models from control engineering can be helpful for determining the capabilities of the present monitoring functions for diagnosis purposes.

\subsection{Behaviour level}

On the behaviour level, the physical phenomena are described which allow a realization of the functions of a technical system. In German design sciences it is proposed (compare e. g. Ehrlenspiel and Meerkamm (2013)) to describe the physical domain by means of elementary physical effects. A relatively small number of these effects (currently about 90 different effects are identified) allows to describe all processes on the behaviour level, thus kind of physical effect chain for an motor encoder of the 
research vehicle is shown in Figure 7.

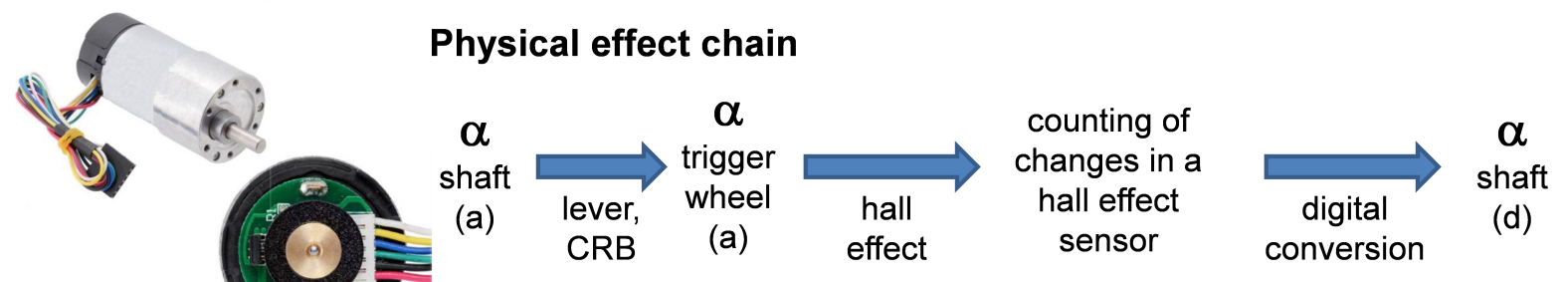

(a): analog; (d): digital; CRB: cohesion of rigid bodies

Figure 7. Physical effect chain motor encoder of the research vehicle

At the beginning on this effect chain, the current angle of the wheel is present at the shaft. The physical effects "lever" and "cohesion of rigid bodies" achieve to transmit the angle to trigger wheel. Based on the Hall Effect, the changes can be counted in a Hall Effect sensor. This allows a digital conversion into digital information concerning the angle of the wheel. This kind of effect chain allows an in-depth analysis of the physical phenomena and their connection and fosters a deeper understanding. Due to the ease of application, physical effects for sensing which have electrical outputs are desirable for monitoring. If one wants to describe the effect chain of a monitoring functionality, it is sensible to use two (pseudo-)effects - the conversion of analogue signals to digital signals and the conversion of digital signals to analogue signals. One very important aspect that could be identified in the sample application is the importance of filters (compare Section 5). Very often mechanical engineers are not aware about the necessity to filter sensor signals, which are very often influenced by noise and distortion. During the design and testing of the research vehicle it became furthermore apparent that only one physical principle for monitoring the surroundings was not sufficient for allowing an orientation in the room as well as a scanning and a mapping of the room. Different physical principles were needed (LIDAR, ultrasonic sensors, odometry sensors and an optical sensor - the camera) need to cooperate in order to fulfil the given task. To conclude, the following insights appear concerning Design for Monitoring in the behaviour level:

- the analysis of physical effect chains can foster a deeper understanding,

- $\quad$ it is sensible to prefer physical effects for sensing which have electrical outputs,

- it is sensible to use pseudo-effects in the physical effect chain to describe analogue/digital conversion,

- the frequent necessity to apply analogue and digital filters to sensors should be considered,

- $\quad$ it is sensible to apply sensors using different physical effects.

\subsection{Structure level}

On the structure level, congruent structures (functional, geometrical, electronic, software), binding and stable interfaces as well as local intelligence are concrete starting points in order to create systems, which ease the realization of monitoring functionality (compare Stetter (2017)). In the design of the research vehicle, one main challenge was the positioning of the sensors. This challenges could be met by an asymmetrical design which takes into consideration the different ranges and necessities of the sensors - the final result is shown in Figure 8.

The research vehicle has seven ultrasonic sensors mounted in the front and two sensors mounted at the back. These sensors detect objects in the range up to $5[\mathrm{~m}]$. They are mainly used to avoid obstacles. On the research vehicle, also a LIDAR sensor is mounted on two servos which allow to move the sensor laterally and vertically. This sensor is used to scan the room. The maximum range of the LIDAR sensor is $40[\mathrm{~m}]$. The conscious sensor placement allowed to fulfil the overall mission (compare Section 5). Additionally it was found that it is very important to assure a stiff mounting of the sensors thus minimizing the effect of vibrations. To conclude, the following insights appear concerning Design for Monitoring in the geometry level:

- the placement and integration of sensors needs to be supported by an in-depth consideration of the specific sensor ranges and other necessities,

- the design of the sensor attachment needs to take into consideration the effects of vibration. 


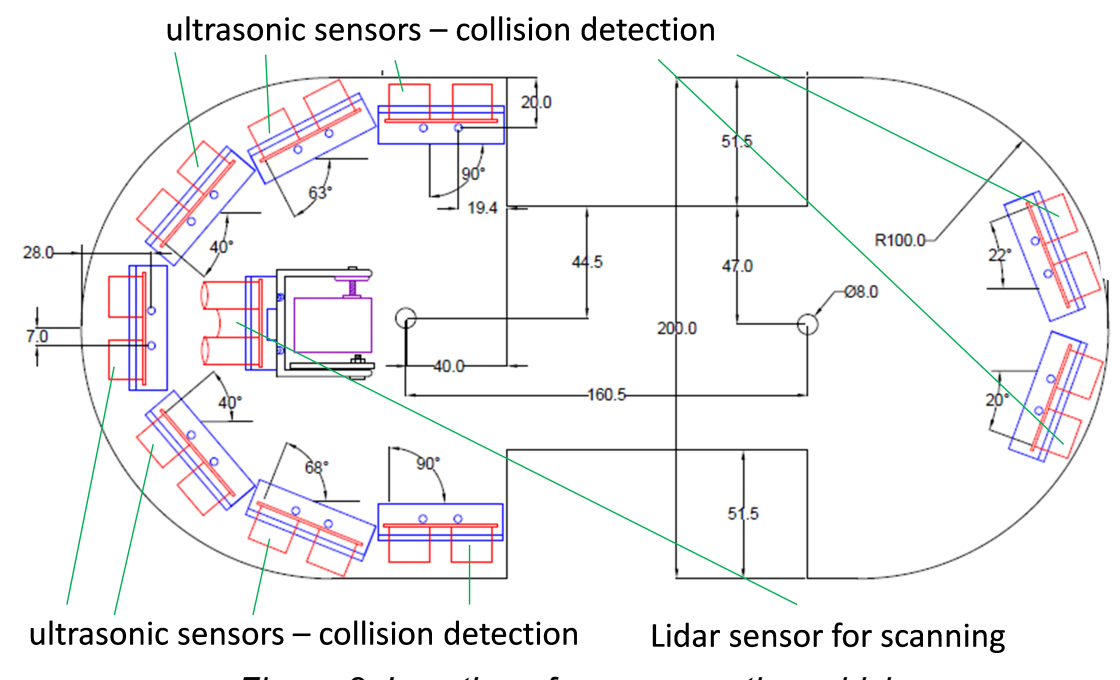

Figure 8. Location of sensors on the vehicle

\subsection{Verification space}

In accordance with many other projects, the application example resulted in the insight that an in-depth verification is one main cornerstone for success. As described for instance in the VDI Guideline 2206, this verification cannot only consist of tests with the complete system, instead all kinds of calculations, simulations and tests need to be carried out with product components, early, unfinished prototypes and initial function samples. In the project, the chassis was the result of a long evolutionary improvement process. The same is true for components which concentrate on monitoring functionality; the position and attachment of the sensors required extensive verification and optimization. To conclude, the following insight appears concerning Design for Monitoring in the verification space:

- monitoring functionalities need to be verified during all phases of the development process on different integration level ranging from early prototypes of single components to the whole product.

\section{APPLICATION EXAMPLE: MAPPING OF A ROOM}

The central purpose of the research vehicle is to foster the development of methods which allow to map unknown environments, meaning the vehicle does not know what size the room is and what is in it before scanning. Therefore, ultrasonic sensors were used to avoid obstacles. The vehicle works as follows:

- In the first stage, the vehicle travels around the room along the wall. If, during the journey, ultrasonic sensors detect an obstacle then the robot turns to the right or left depending on which side the object is on.

- After a time span declared by the user, the vehicle stops and makes a room scan.

- The results of scanning are saved on the microSD card and the vehicle returns to follow the wall.

- After receiving the finish command, the vehicle stops and saves the final data of its work. Until then, the robot drives and maps the room.

The task of the AGV was to scan the room that is presented in Figure 9.

The results of the mapping were compared with the actual dimensions of the room shown in Figure 9. All dimensions of the room are given in centimetres. The room has obstacles such as litter bins, heaters by the walls and chairs in the middle of the room. Another difficulty for the AGV was the shape of the room, in which there were, for example, doors from other rooms and a column in the middle of the room. Figure 10 presents the initial result of mapping the room by the AGV. The vehicle scanned the room 16 times. It can be seen that the overall shape of the map is similar to the model shown in Figure 9. Unfortunately, many measurements do not overlap with places where there are probably walls, therefore for a more accurate comparison, the mapping result was compared to the actual dimensions of the room (Figure 10 - right side). The black colour indicates the result of mapping, while the green colour shows the actual dimensions of the room. In addition, examples of wrong measurements are marked in red. The figure shows that most measurements coincide with the actual dimensions of the walls of the room. However, there are also many points on the map that do not coincide with the walls. The number 5 

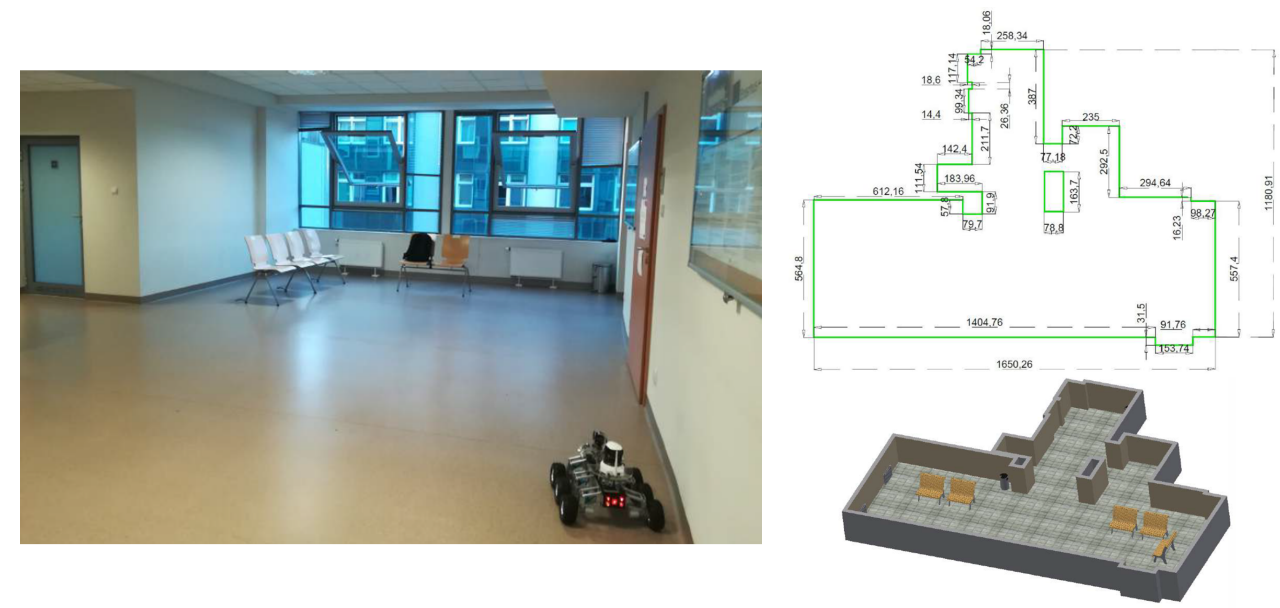

Figure 9. Application example: room to be mapped

indicates the place where there are the transparent glass doors. Although they were closed, the laser sensor measured the objects behind them, which were marked with number 1 . Areas with the largest errors in measurements were marked with the number 3 , where the difference between the measurement and the actual location of the walls exceeds even $150[\mathrm{~cm}]$. However, these measurements are outside the room, so in the case of navigation they are not as troublesome as the noises located inside of the room. Examples of this measurements are marked with number 2. On the other hand, areas with number 4 show the measurements of standing chairs in the room. It can be observed that points between the chairs and the wall are included, which do not exist in reality. In order to improve the quality of the map, it was decided to apply a filtration of measurements indicating the location of the walls of the room; Figure 11 presents the result of the filtration of measurements indicating the location of the walls.
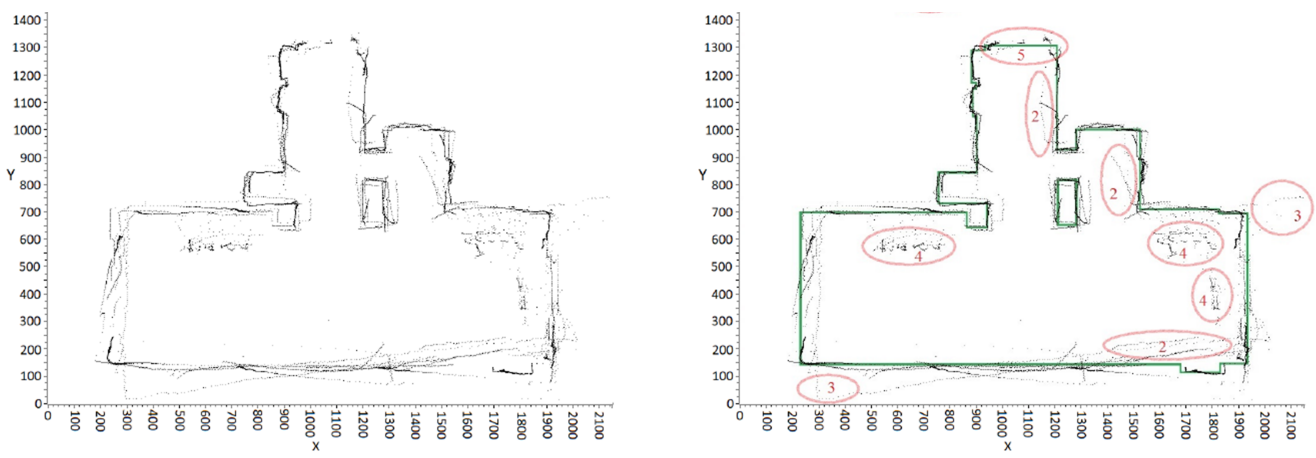

Figure 10. Initial mapping result
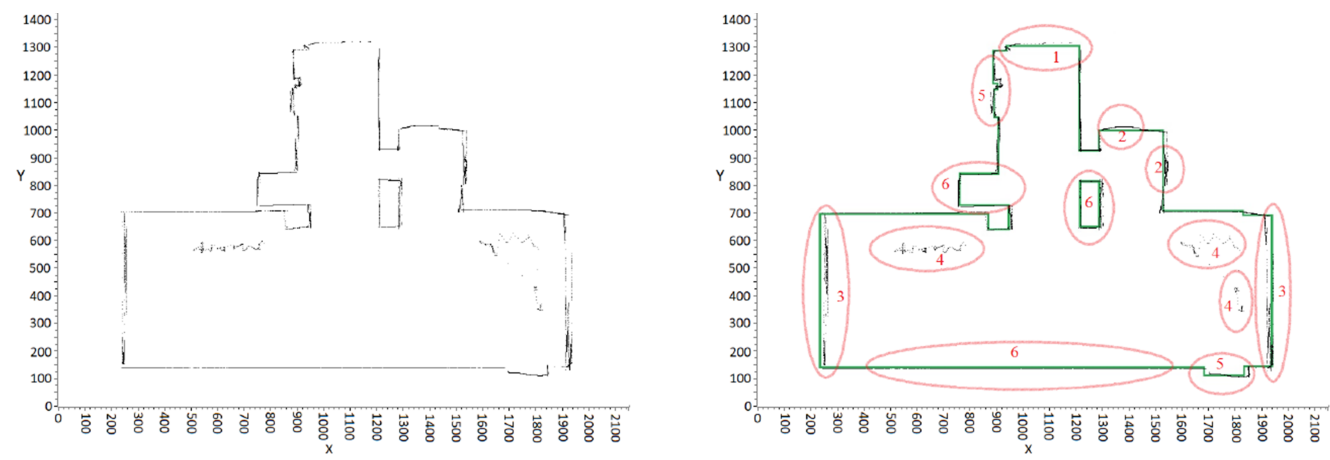

Figure 11. Mapping result after filtration

A Savitzky and Golay filter was used (Savitzky and Golay (1964)). The filtration was applied separately for each wall in the room, that is, for a total of 36 walls. It can be seen that most of the walls have been 
smoothed out and evened out. The walls are not perfectly even, but the results after filtration are much more accurate. This underlines the monitoring effectiveness of the developed design.

\section{SUMMARY}

The objective of this paper is the presentation of insights concerning design, which can ease to realization of monitoring functionality. The main benefit of this paper are these insights, which are summarized under the notion "Design for Monitoring". They were generated by means of a detailed investigation of a sample project - the design of a research vehicle which is intended to foster the development of mapping systems and algorithms. The investigation was structured based on a model of product concretization. The product exhibits a convincing monitoring performance. Until now the presented research is based on a small number of different design processes and an in-depth literature analysis. Future research is needed to expand the insights to other types of technical systems.

\section{REFERENCES}

Åström, K. J. and Wittenmark, B. (2013), “Adaptive Control” Courier Corporation.

Blanke, M., Kinnaert M., Lunze, J. and Staroswiecki, M. (2016), "Diagnosis and fault-tolerant control", Springer.

Carryer, J. E., Ohline R. M. and Kenny, T.W.. (2010), "Introduction to mechatronic design", Pearson.

Cherdantseva, Y., Burnap, P., Blyth, A., Eden, P., Jones, K., Soulsby, H. and Stoddart. K. (2016), "A review of cyber security risk assessment methods for scada systems", Computers \& security, Vol. 56, pp. 1-27.

Ehrlenspiel, K. and Meerkamm, H. (2013), "Integrierte Produktentwicklung: Denkabläufe, Methodeneinsatz, Zusammenarbeit", Hanser, München.

Eigner, M., Dickkopf, T., and Apostolov, H. (2018), "Interdsziplinäre Konstruktionsmethoden und - prozesse zur Entwicklung cybertronischer Produkte - Teil 1", Konstruktion, pp. 84-90.

Eisenbart, B., Gericke, K., Blessing, T.M. and McAloone, T.C. (2017), "A dsm-based framework for integrated function modelling: concept, application and evaluation", Research in Engineering Design, Vol. 28, pp. 25-51.

Frisk, E. and Krysander, M. (2008), "Sensor placement for maximum fault isolability", IEEE Transactions on Systems, Man, and Cybernetics - Part A: Systems and Humans, Vol. 38, No. 6, pp. 1398-1410.

Holder, K., Zech, A., Ramsaier, M., Stetter, R., Niedermeier, H.-P., Rudolph, S. and Till, M. (2017), "Model-based requirements management in gear systems design based on graph-based design languages", Applied Sciences, Vol. 7, No. 11, p. 1112.

Hruschka, P. (2019), "Business analysis und requirements engineering: Produkte und Prozesse nachhaltig verbessern", Carl Hanser Verlag GmbH Co KG.

Isermann, R. (2006), "Fault-diagnosis systems: an introduction from fault detection to fault tolerance", Springer Science \& Business Media.

Korbicz, J., Koscielny, J.M, Kowalczuk, Z. and Cholewa, W. (2012), "Fault diagnosis: models, artificial intelligence, applications", Springer Science \& Business Media.

Livotov, P. and Petrov, V. (2011), "TRIZ: Innovation and Inventive Problem Solving", TriS Europe.

Pfenning, M. (2017), "Durchgängiges Engineering durch die Integration von PLM und MBSE", Technische Universität Kaiserslautern.

Ponn, J. and Lindemann, U. (2011), "Konzeptentwicklung und Gestaltung technischer Produkte: systematisch von Anforderungen zu Konzepten und Gestaltlösungen", Springer-Verlag.

Savitzky, A. and Golay, M.J.E. (1964), "Smoothing and differentiation of data by simplified least squares procedures", Analytical chemistry, Vol. 36, No. 8, pp. 1627-1639.

Stetter, R. (2017), "Monitoring in product development", In: Conference proceedings of the 14th european workshop on advanced control and diagnosis $(A C D)$.

Stetter, R. (2019), "Fault-Tolerant Design and Control of Automated Vehicles and Processes", Springer.

Stetter, R. and Phleps, U. (2011), "Design for diagnosis", In: DS 68-5: Proceedings of the 18th International Conference on Engineering Design (ICED 11), Vol. 5, pp. 91-102.

Van Long, D.O., Fillatre, L. and Nikiforov, I. (2015), "Sequential monitoring of scada systems against cyber/physical attacks", IFAC-PapersOnLine, Vol. 48, No. 21, pp.746-753.

Witczak, M. (2014), "Fault diagnosis and fault-tolerant control strategies for non-linear systems", Springer.

\section{ACKNOWLEDGEMENTS}

The work was supported by the National Science Centre, Poland under Grant: UMO-2017/27/ B/ST7/00620. 